\title{
An analytical verification test for numerically simulated convective flow above a thermally heterogeneous surface
}

\author{
A. Shapiro ${ }^{1,2}$, E. Fedorovich ${ }^{1}$, and J. A. Gibbs ${ }^{1}$ \\ ${ }^{1}$ School of Meteorology, University of Oklahoma, Norman, OK, USA \\ ${ }^{2}$ Center for Analysis and Prediction of Storms, University of Oklahoma, Norman, OK, USA \\ Correspondence to: A. Shapiro (ashapiro@ou.edu)
}

Received: 07 January 2015 - Published in Geosci. Model Dev. Discuss.: 16 March 2015

Revised: 27 May 2015 - Accepted: 02 June 2015 - Published: 18 June 2015

\begin{abstract}
An analytical solution of the Boussinesq equations for the motion of a viscous stably stratified fluid driven by a surface thermal forcing with large horizontal gradients (step changes) is obtained. This analytical solution is one of the few available for wall-bounded buoyancy-driven flows. The solution can be used to verify that computer codes for Boussinesq fluid system simulations are free of errors in formulation of wall boundary conditions and to evaluate the relative performances of competing numerical algorithms. Because the solution pertains to flows driven by a surface thermal forcing, one of its main applications may be for testing the no-slip, impermeable wall boundary conditions for the pressure Poisson equation. Examples of such tests are presented.
\end{abstract}

\section{Introduction}

Thermal disturbances associated with variations in underlying surface properties can drive local circulations in the atmospheric boundary layer (Atkinson, 1981; Briggs, 1988; Hadfield et al., 1991; Segal and Arritt, 1992; Simpson, 1994; Mahrt et al., 1994; Pielke, 2001; McPherson, 2007; Kang et al., 2012) and affect the development of the convective boundary layer (Patton et al., 2005; van Heerwaarden et al., 2014). Computational fluid dynamics (CFD) codes for modelling such flows commonly solve the Boussinesq equations of motion and thermal energy for a viscous/diffusive stably stratified fluid. In this paper we present an analytical solution of the Boussinesq equations for flows driven by a surface thermal forcing with large gradients (step changes) in the horizontal. The solution can be used to verify that CFD codes for Boussinesq fluid system simulations are free of errors, and to evaluate the relative performances of competing numerical algorithms. Such verification procedures are important in the development of CFD models designed for research, operational, and classroom applications.

We solve the linearized Navier-Stokes and thermal energy equations analytically for the case where the surface buoyancy varies laterally as a square wave (Fig. 1). Attention is restricted to the steady state. No boundary-layer approximations are made; the solution is non-hydrostatic, and both horizontal and vertical derivatives are included in the viscous stress and thermal diffusion terms. The solution is similar to that of Axelsen et al. (2010) for katabatic flow above a cold strip but is easier to evaluate (no slope present) and applies to the more general scenario where the viscosity and diffusivity coefficients can differ. The flow is also similar to a special case (no slope) considered by Egger (1981), although a final analytical solution was not provided in that study. Strictly speaking, the linearized Navier-Stokes equations apply to a class of very low Reynolds number motions known as creeping flows. Such flows appear in studies of lubrication, locomotion of microorganisms, lava flow, and flow in porous media. Of course, for the task at hand, if our linear solution is to serve as a benchmark for a nonlinear numerical model solution, it is essential that the parameter space be restricted to values for which the model's nonlinear terms are negligible.

Because the solution pertains to flows driven by a surface thermal forcing, one of its main applications may be as a test for surface boundary conditions in the pressure Poisson equation. In models of atmospheric boundary-layer flows, the buoyancy is a major contributor to the forcing term in the Poisson equation and also appears in the associated 


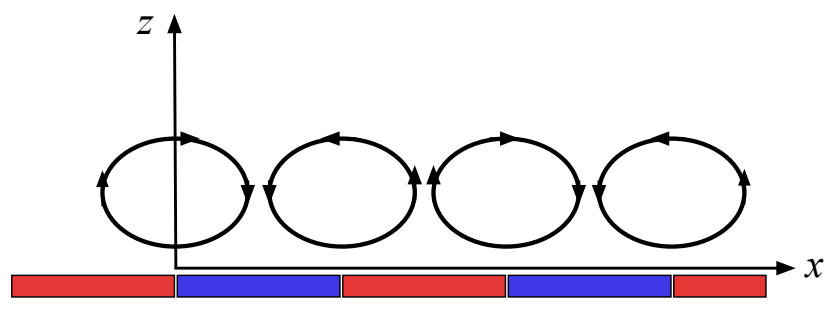

Figure 1. Schematic of two-dimensional $(x, z)$ thermal convection induced by a surface buoyancy that varies horizontally $(x)$ as a square wave. Red denotes positive surface buoyancy, blue denotes negative surface buoyancy.

surface boundary condition. The pressure boundary condition on a solid boundary in incompressible (Boussinesq) fluid flows is an important and complex issue that has long been fraught with technical difficulties and controversies (Strikwerda, 1984; Orszag et al., 1986; Gresho and Sani, 1987; Gresho, 1990; Temam, 1991; Henshaw, 1994; Petersson, 2001; Sani et al., 2006; Rempfer, 2006; Guermond et al., 2006; Nordström et al., 2007; Shirokoff and Rosales, 2011; Hosseini and Feng, 2011; Vreman, 2014). Typical fractional-step solution methodologies and associated pressure (or pseudo-pressure) boundary-condition implementations are often verified using various prototypic flows such as Poiseuille flows, lid-driven cavity flows, flows over cylinders or bluff bodies, viscously decaying vortices, and dam-break flows. We are unaware of verification tests in which flows were driven by a heterogeneous surface buoyancy forcing. Our solution is designed to fill this gap.

The analytical solution is derived in Sect. 2. In Sect. 3, this solution is compared to numerically simulated fields in a steady state. Two versions of a numerical code are run: a version in which the correct surface pressure boundary condition is applied, and a version in which the pressure condition is mis-specified. A summary follows in Sect. 4.

\section{Analytical solution}

We derive the solution for steady flow over an underlying surface along which the buoyancy varies laterally as a single-harmonic function. This single-harmonic solution is then used as a building block in a Fourier representation of the square-wave solution.

\subsection{Governing equations}

Consider the flow of a viscous stably stratified fluid that fills the semi-infinite domain above a solid horizontal surface (placed at $z=0$ ). This surface undergoes a steady thermal forcing that varies periodically in the right-hand Cartesian $x$ direction but is independent of the $y$ direction. The two-dimensional $(x, z)$ flow is periodic in $x$ and satisfies the linearized (assuming the disturbance is of small amplitude) governing equations under the Boussinesq approximation,

$$
\begin{aligned}
& 0=-\frac{\partial \Pi}{\partial x}+v \nabla^{2} u, \\
& 0=-\frac{\partial \Pi}{\partial z}+b+v \nabla^{2} w, \\
& 0=-N^{2} w+\alpha \nabla^{2} b, \\
& \frac{\partial u}{\partial x}+\frac{\partial w}{\partial z}=0 .
\end{aligned}
$$

Apart from notational differences, Eqs. (1)-(4) are the two-dimensional steady state versions of Eqs. (55)-(57) of Sect. II of Chandrasekhar (1961). Equations (1) and (2) are the horizontal $(x)$ and vertical $(z)$ equations of motion, respectively, Eq. (3) is the thermal energy equation (differential form of the first law of thermodynamics) expressed in terms of the buoyancy variable (defined below), and Eq. (4) is the incompressibility condition. Here $u$ and $w$ are the horizontal and vertical velocity components, $\Pi \equiv\left[p-p_{\mathrm{e}}(z)\right] / \rho_{\mathrm{w}}$ is the kinematic pressure perturbation $\left[p\right.$ is pressure, $p_{\mathrm{e}}(z)$ is pressure in a hydrostatic environmental state in which the density profile is $\rho_{\mathrm{e}}(z), \rho_{\mathrm{w}}$ is a constant reference density, say, $\left.\rho_{\mathrm{e}}(0)\right]$, and $b \equiv-g\left[\rho-\rho_{\mathrm{e}}(z)\right] / \rho_{\mathrm{W}}$ is the buoyancy, where $\rho$ is the actual density, and $g$ is the acceleration due to gravity. The Brunt-Väisälä frequency $N \equiv \sqrt{-\left(g / \rho_{\mathrm{w}}\right) \mathrm{d} \rho_{\mathrm{e}} / \mathrm{d} z}$ of the ambient fluid (Kundu, 1990), kinematic viscosity $v$, and thermal diffusivity $\alpha$ are taken constant.

We obtain our solution using a standard vorticity/streamfunction formulation. Cross-differentiating Eqs. (1) and (2) yields the vorticity equation,

$0=-\frac{\partial b}{\partial x}+v \nabla^{2} \eta$

where $\eta \equiv \partial u / \partial z-\partial w / \partial x$ is the vorticity. Eliminating $b$ from Eqs. (3) and (5) yields

$\nabla^{4} \eta=\frac{N^{2}}{\nu \alpha} \frac{\partial w}{\partial x}$.

Introducing a streamfunction $\psi$ defined through

$u=\partial \psi / \partial z, \quad w=-\partial \psi / \partial x$,

guarantees that Eq. (4) is satisfied and transforms Eq. (6) into a single equation for $\psi$,

$\nabla^{6} \psi+\frac{N^{2}}{v \alpha} \frac{\partial^{2} \psi}{\partial x^{2}}=0$.

The dependent variables are assumed to vanish far above the surface $(z \rightarrow \infty)$. On the surface we apply no-slip $(u=0)$ and impermeability $(w=0)$ conditions, and specify a periodic (in $x$ ) buoyancy distribution. As we will now see, restricting the dependent variables to steady periodic forms that vanish as $z \rightarrow \infty$ also restricts acceptable distributions of the surface buoyancy. The restriction was first noted by Egger 
(1981, Sect. 3c), though without details. Averaging Eq. (3) over one period (using $w=-\partial \psi / \partial x$ ) yields $d^{2} \bar{b} / \mathrm{d} z^{2}=0$, which integrates to $\bar{b}=A+B z$ ( $\bar{b}$ is the average of $b ; A$ and $B$ are constants). Taking $b \rightarrow 0$ as $z \rightarrow \infty$, implies that $\bar{b} \rightarrow 0$ as $z \rightarrow \infty$, in which case $A=B=0$, and $\bar{b}(z)=0$. In particular, at the surface, $\bar{b}(0)=0$. If a surface distribution $b(x, 0)$ violates this condition, the ground acts as a net heat source/sink. In an unsteady model, such a source/sink would force a continually upward-developing disturbance and a steady state could never be attained.

\subsection{Single-harmonic forcing}

For a surface buoyancy of the form $b(x, 0) \propto \sin k x$, Eq. (3) indicates that $\psi$ is of the form

$\psi=A(z) \cos k x$.

Application of Eq. (9) in Eq. (8) yields

$\left(\frac{d^{2}}{\mathrm{~d} z^{2}}-k^{2}\right)^{3} A-\frac{N^{2} k^{2}}{v \alpha} A=0$

which has solutions of the form $A \propto e^{M z}$ for $M$ satisfying

$\left(M^{2}-k^{2}\right)^{3}=\frac{N^{2} k^{2}}{\nu \alpha}$.

Taking the one-third power of Eq. (11) yields a useful intermediate result:

$M^{2}-k^{2}=\frac{N^{2 / 3} k^{2 / 3}}{v^{1 / 3} \alpha^{1 / 3}} e^{2 n \pi i / 3}$,

where $n$ is an integer. Rearranging Eq. (12) and taking the square root yields

$M= \pm \sqrt{k^{2}+\frac{N^{2 / 3} k^{2 / 3}}{v^{1 / 3} \alpha^{1 / 3}} e^{2 n \pi i / 3}}$.

Equation (13) furnishes six roots, two for each of $n=0,1,2$. To ensure that $A(z) \rightarrow 0$ as $z \rightarrow \infty$, we reject the roots with a positive real part. With the radicand of Eq. (13) expressed in polar form, the physically acceptable roots are

$$
\begin{aligned}
& M_{0}=-\sqrt{k^{2}+\frac{N^{2 / 3} k^{2 / 3}}{v^{1 / 3} \alpha^{1 / 3}}, \quad(n=0),} \\
& M_{1}=-r^{1 / 2} e^{i \phi / 2}, \quad(n=1), \\
& M_{2}=-r^{1 / 2} e^{-i \phi / 2}, \quad(n=2),
\end{aligned}
$$

where the subscript on $M$ denotes the associated value of $n$, and $r$ and $\phi$ are defined by

$$
r \equiv \sqrt{\left[k^{2}+\frac{N^{2 / 3} k^{2 / 3}}{v^{1 / 3} \alpha^{1 / 3}} \cos \left(\frac{2 \pi}{3}\right)\right]^{2}+\left[\frac{N^{2 / 3} k^{2 / 3}}{v^{1 / 3} \alpha^{1 / 3}} \sin \left(\frac{2 \pi}{3}\right)\right]^{2}},
$$

$$
\begin{aligned}
& \cos \phi=\frac{1}{r}\left[k^{2}+\frac{N^{2 / 3} k^{2 / 3}}{v^{1 / 3} \alpha^{1 / 3}} \cos \left(\frac{2 \pi}{3}\right)\right], \\
& \sin \phi=\frac{1}{r}\left(\frac{N^{2 / 3} k^{2 / 3}}{v^{1 / 3} \alpha^{1 / 3}}\right) \sin \left(\frac{2 \pi}{3}\right)>0 .
\end{aligned}
$$

While solving Eq. (16) for $\phi$, care must be taken when evaluating arcsin or arccos functions that $\phi$ appears in the correct quadrant ( $\phi$ should be in quadrant I or II so $\phi / 2$ should always be in quadrant I). Also note from Eq. (14b) and (14c) that $M_{2}$ is the complex conjugate of $M_{1}\left(M_{2}=M_{1}^{*}\right)$, a fact that will often be used below.

With the general solution for $\psi$ written as

$$
\psi=\left(B e^{M_{0} z}+C e^{M_{1} z}+D e^{M_{2} z}\right) \cos k x,
$$

where $B, C$, and $D$ are constants, the vorticity becomes,

$$
\begin{aligned}
\eta & =\left[B\left(M_{0}^{2}-k^{2}\right) e^{M_{0} z}+C\left(M_{1}^{2}-k^{2}\right) e^{M_{1} z}\right. \\
& \left.+D\left(M_{2}^{2}-k^{2}\right) e^{M_{2} z}\right] \cos k x,
\end{aligned}
$$

and the buoyancy follows from Eq. (3) as

$$
\begin{aligned}
b & =\frac{k N^{2}}{\alpha}\left(\frac{B}{M_{0}^{2}-k^{2}} e^{M_{0} z}+\frac{C}{M_{1}^{2}-k^{2}} e^{M_{1} z}\right. \\
& \left.+\frac{D}{M_{2}^{2}-k^{2}} e^{M_{2} z}\right) \sin k x+b_{h},
\end{aligned}
$$

where $\nabla^{2} b_{h}=0$. In view of Eq. (12), Eq. (19) becomes

$$
\begin{aligned}
b & =\frac{k^{1 / 3} v^{1 / 3} N^{4 / 3}}{\alpha^{2 / 3}}\left(B e^{M_{0} z}+e^{-2 \pi i / 3} C e^{M_{1} z}\right. \\
& \left.+e^{-4 \pi i / 3} D e^{M_{2} z}\right) \sin k x+b_{\mathrm{h}} .
\end{aligned}
$$

Applying Eqs. (18) and (20) in Eq. (5) yields an equation for $\partial b_{\mathrm{h}} / \partial x$ which, upon use of Eq. (12) and $M_{2}=M_{1}^{*}$, reduces to $\partial b_{\mathrm{h}} / \partial x=0$. So $b_{\mathrm{h}}$ is, at most, a function of $z$. Since $\nabla^{2} b_{\mathrm{h}}=0, b_{\mathrm{h}}$ is, at most, a linear function of $z$, and since $b$ should vanish as $z \rightarrow \infty$, that linear function must be 0 . Thus, $b_{\mathrm{h}}=0$.

The pressure follows from Eqs. (1) and (12) as

$$
\begin{aligned}
\Pi & =\frac{v^{2 / 3} N^{2 / 3}}{k^{1 / 3} \alpha^{1 / 3}}\left(B M_{0} e^{M_{0} z}+C M_{1} e^{2 \pi i / 3} e^{M_{1} z}\right. \\
& \left.+D M_{2} e^{4 \pi i / 3} e^{M_{2} z}\right) \sin k x+G(z),
\end{aligned}
$$

where $G(z)$ is a function of integration. Applying Eq. (21) in Eq. (2), and using Eq. (11) yields $\mathrm{d} G / \mathrm{d} z=0$, so $G$ is constant. For $\Pi$ to vanish as $z \rightarrow \infty$, this constant must be 0 .

The surface conditions determine $B, C$, and $D$. The surface buoyancy is

$b(x, 0)=b_{0} \sin k x$,

where $b_{0}$ is a constant forcing amplitude. Application of Eq. (20) in Eq. (22) yields

$$
B+e^{-2 \pi i / 3} C+e^{-4 \pi i / 3} D=\frac{b_{0} \alpha^{2 / 3}}{k^{1 / 3} v^{1 / 3} N^{4 / 3}} .
$$


In view of Eqs. (7) and (17), the impermeability condition $w(x, 0)=0$ and no-slip condition $u(x, 0)=0$ yield

$B+C+D=0$,

$B M_{0}+C M_{1}+D M_{2}=0$.

Straightforward but lengthy manipulations yield the solution of Eqs. (23)-(25):

$B=-\left(\frac{b_{0} \alpha^{2 / 3}}{\sqrt{3} k^{1 / 3} v^{1 / 3} N^{4 / 3}}\right) \frac{2 r^{1 / 2} \sin (\phi / 2)}{M_{0}+2 r^{1 / 2} \cos (\pi / 3+\phi / 2)}$,

$C=-i\left(\frac{b_{0} \alpha^{2 / 3}}{\sqrt{3} k^{1 / 3} v^{1 / 3} N^{4 / 3}}\right) \frac{M_{2}-M_{0}}{M_{0}+2 r^{1 / 2} \cos (\pi / 3+\phi / 2)}$,

$D=i\left(\frac{b_{0} \alpha^{2 / 3}}{\sqrt{3} k^{1 / 3} v^{1 / 3} N^{4 / 3}}\right) \frac{M_{1}-M_{0}}{M_{0}+2 r^{1 / 2} \cos (\pi / 3+\phi / 2)}$.

Applying Eqs. (26)-(28) in Eqs. (17), (20), and (18), with Eq. (12) used in the latter equation, and noting that $B$ is real, while $D=C^{*}$ (since $M_{2}=M_{1}^{*}$ ), we obtain

$$
\begin{aligned}
& b=\frac{2 b_{0}}{\sqrt{3}} \\
& \frac{e^{-Z_{\mathrm{c}}}\left[\mu \cos \left(Z_{\mathrm{s}}+\pi / 6\right)+\cos \left(Z_{\mathrm{s}}+\pi / 6+\phi / 2\right)\right]-e^{M_{0} z} \sin (\phi / 2)}{\mu+2 \cos (\pi / 3+\phi / 2)} \sin k x,
\end{aligned}
$$

$$
\begin{aligned}
& \psi=\frac{2 b_{0} \alpha^{2 / 3}}{\sqrt{3} k^{1 / 3} v^{1 / 3} N^{4 / 3}} \\
& \frac{e^{-Z_{\mathrm{c}}}\left[\mu \sin Z_{\mathrm{s}}+\sin \left(Z_{\mathrm{s}}+\phi / 2\right)\right]-e^{M_{0} z} \sin (\phi / 2)}{\mu+2 \cos (\pi / 3+\phi / 2)} \cos k x,
\end{aligned}
$$

where

$$
Z_{\mathrm{s}} \equiv z r^{1 / 2} \sin (\phi / 2), \quad Z_{\mathrm{c}} \equiv z r^{1 / 2} \cos (\phi / 2), \quad \mu \equiv M_{0} / r^{1 / 2} .
$$

Application of Eq. (30) in Eq. (7) yields the velocity components as

$$
\begin{aligned}
& u=\frac{2 b_{0} \alpha^{2 / 3} r^{1 / 2}}{\sqrt{3} k^{1 / 3} v^{1 / 3} N^{4 / 3}} \\
& \frac{e^{-Z_{\mathrm{c}}}\left[\mu \sin \left(\phi / 2-Z_{\mathrm{s}}\right)-\sin Z_{\mathrm{s}}\right]-\mu e^{M_{0} z} \sin (\phi / 2)}{\mu+2 \cos (\pi / 3+\phi / 2)} \cos k x,
\end{aligned}
$$

$$
\begin{aligned}
& w=\frac{2 b_{0} \alpha^{2 / 3} k^{2 / 3}}{\sqrt{3} v^{1 / 3} N^{4 / 3}} \\
& \frac{e^{-Z_{\mathrm{c}}}\left[\mu \sin Z_{\mathrm{s}}+\sin \left(Z_{\mathrm{s}}+\phi / 2\right)\right]-e^{M_{0} z} \sin (\phi / 2)}{\mu+2 \cos (\pi / 3+\phi / 2)} \sin k x .
\end{aligned}
$$

\subsection{Piecewise constant (square wave) forcing}

Next, consider the case where the surface buoyancy varies horizontally as a square wave, with a distribution over one period $L$ given by

$b(x, 0)= \begin{cases}b_{\max }, & 0<x<L / 2, \\ -b_{\max }, & L / 2<x<L .\end{cases}$

Such a distribution can be expressed as the Fourier series:

$b(x, 0)=\sum_{n=1}^{\infty} b_{n} \sin \left(\frac{n \pi x}{L}\right)$,

$b_{n}=\frac{2}{L} \int_{0}^{L} b(x, 0) \sin \left(\frac{n \pi x}{L}\right)$.

Application of Eq. (34) in Eq. (36) yields

$b_{n}=\frac{2 b_{\max }}{n \pi}[1-2 \cos (n \pi / 2)+\cos (n \pi)]$.

The solutions for $b, \psi, u$, and $w$ can then be written as summations over the single-harmonic solutions (29), (30), (32), and (33), with $k$ related to $n$ by

$k=\frac{n \pi}{L}$,

and with $b_{0}$ replaced by $b_{n}$ :

$$
\begin{aligned}
b & =\frac{2}{\sqrt{3}} \sum_{n=1}^{\infty} b_{n} \\
& \frac{e^{-Z_{\mathrm{c}}}\left[\mu \cos \left(Z_{\mathrm{s}}+\pi / 6\right)+\cos \left(Z_{\mathrm{s}}+\pi / 6+\phi / 2\right)\right]-e^{M_{0} z} \sin (\phi / 2)}{\mu+2 \cos (\pi / 3+\phi / 2)} \\
& \sin \left(\frac{n \pi x}{L}\right),
\end{aligned}
$$

$$
\begin{aligned}
& \psi=\frac{2 \alpha^{2 / 3}}{\sqrt{3} v^{1 / 3} N^{4 / 3}} \sum_{n=1}^{\infty} \frac{b_{n}}{k^{1 / 3}} \\
& \frac{e^{-Z_{\mathrm{c}}}\left[\mu \sin Z_{\mathrm{s}}+\sin \left(Z_{\mathrm{s}}+\phi / 2\right)\right]-e^{M_{0} z} \sin (\phi / 2)}{\mu+2 \cos (\pi / 3+\phi / 2)} \cos \left(\frac{n \pi x}{L}\right),
\end{aligned}
$$

$$
\begin{aligned}
& u=\frac{2 \alpha^{2 / 3}}{\sqrt{3} v^{1 / 3} N^{4 / 3}} \sum_{n=1}^{\infty} b_{n} \frac{r^{1 / 2}}{k^{1 / 3}} \\
& \frac{e^{-Z_{\mathrm{c}}}\left[\mu \sin \left(\phi / 2-Z_{\mathrm{s}}\right)-\sin Z_{\mathrm{s}}\right]-\mu e^{M_{0} z} \sin (\phi / 2)}{\mu+2 \cos (\pi / 3+\phi / 2)} \cos \left(\frac{n \pi x}{L}\right),
\end{aligned}
$$

$$
\begin{aligned}
& w=\frac{2 \alpha^{2 / 3}}{\sqrt{3} v^{1 / 3} N^{4 / 3}} \sum_{n=1}^{\infty} b_{n} k^{2 / 3} \\
& \frac{e^{-Z_{c}}\left[\mu \sin Z_{\mathrm{s}}+\sin \left(Z_{\mathrm{s}}+\phi / 2\right)\right]-e^{M_{0} z} \sin (\phi / 2)}{\mu+2 \cos (\pi / 3+\phi / 2)} \sin \left(\frac{n \pi x}{L}\right) .
\end{aligned}
$$




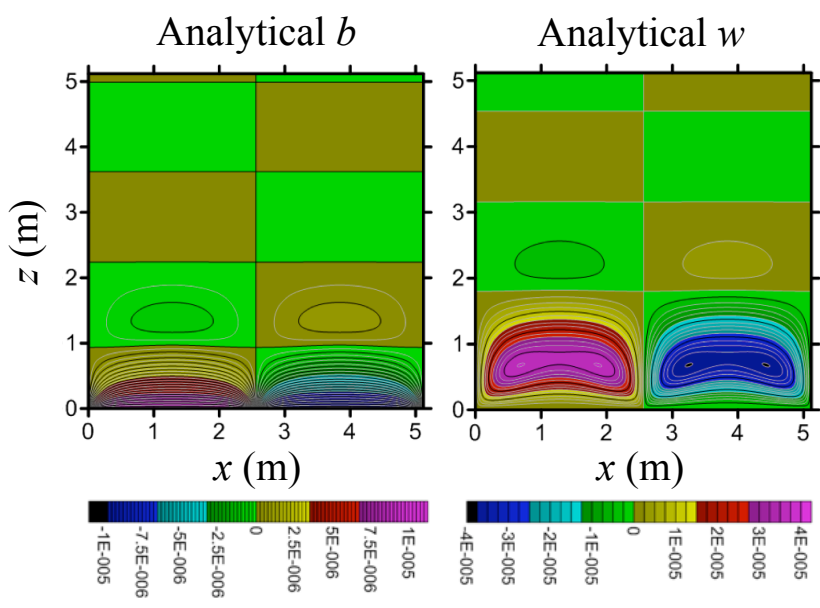

Figure 2. Vertical cross section of the analytical (A-1) buoyancy $b$ and vertical velocity $w$ fields from the first test case. Colour bar units are $\mathrm{ms}^{-2}$ for $b$, and $\mathrm{ms}^{-1}$ for $w$.

\section{Verification tests}

A solution of the linearized equations may be used to verify a nonlinear code if the nonlinear terms are sufficiently small. Unfortunately, a priori estimates of such terms expressed, for example, through a Reynolds number, are not straightforward since the relevant velocity and length scales in our problem are only evident after a solution has been obtained. We thus seek an appropriate set of test parameters through trial and error, guided by a posteriori linear solution estimates of the terms $\boldsymbol{u} \cdot \nabla b$ and $\boldsymbol{u} \cdot \nabla \eta[\boldsymbol{u}=(u, w)]$ present in nonlinear versions of Eqs. (3) and (5), respectively. Specifically, for any computed candidate solution, we formed the ratios of the largest values of those nonlinear terms to the largest values of the corresponding linear terms, that is, the terms actually present in Eqs. (3) and (5). We need only consider one such linear term per ratio since Eqs. (3) and (5) are comprised of two terms of equal magnitude. A solution was deemed to be sufficiently linear if

$R_{\eta} \equiv \frac{\max |\boldsymbol{u} \cdot \nabla \eta|}{\max |\partial b / \partial x|}<\varepsilon$, and $R_{b} \equiv \frac{\max |\boldsymbol{u} \cdot \nabla b|}{\max \left|\alpha \nabla^{2} b\right|}<\varepsilon$,

where $\varepsilon(\ll 1)$ is a prescribed threshold. The suitability of this approach was confirmed by the very close agreement between the analytical solutions and the numerical solutions obtained with the correct surface pressure condition.

The numerical model employed in our tests is a variant of a direct numerical simulation (DNS) code used in the boundary-layer and slope-flow studies of Fedorovich et al. (2001), Fedorovich and Shapiro (2009a, b), and Shapiro and Fedorovich $(2013,2014)$. The model solves the Boussinesq governing equations on a staggered (Arakawa $\mathrm{C}$ ) grid. Although designed for three-dimensional simulations, the model was run in a two-dimensional $(x, z)$ mode. The overall solution procedure is patterned on a fractional step method proposed by Chorin (1968). In our version, the prognostic equations are integrated using a filtered leapfrog scheme with explicit treatment of the viscous term. The pressure is diagnosed from a Poisson equation (Eq. A3b, discussed in the Appendix), which is solved using a fast Fourier transform technique in horizontal planes, and a tridiagonal matrix inversion in the vertical. The surface condition on pressure is the inhomogeneous Neumann condition (INC) that arises from projecting the vertical equation of motion into the vertical and imposing the impermeability condition (Vreman, 2014; also see the Appendix). We also run a version of the code in which the surface pressure condition is mis-specified as a homogeneous Neumann condition (HNC). We hasten to add, however, that our implementation of the HNC may be quite different from implementations described in the literature. We elaborate on these technical differences and review general aspects of the problem of surface pressure specification in the Appendix.

The analytical solution was evaluated on an un-staggered $(x, z)$ grid extending over one period of the square wave $(x=0$ to $x=L)$. The series were truncated at 50000 terms. The governing parameters were adjusted so that the linearity criteria were satisfied in comparisons with $\varepsilon=5 \times 10^{-3}$.

In the first test, we set $v=\alpha=0.001 \mathrm{~m}^{2} \mathrm{~s}^{-1}, N=$ $0.02 \mathrm{~s}^{-1}, L=5.12 \mathrm{~m}$, and $b_{\max }=1 \times 10^{-5} \mathrm{~m} \mathrm{~s}^{-2}$. For the analytical solution A-1, the $(x, z)$ grid consisted of 513 points in the $x$ direction and 1025 points in the $z$ direction, with grid spacings $\Delta x=\Delta z=0.01 \mathrm{~m}$. The linearity criteria (Eq. 43) were satisfied with $R_{\eta} \cong 8.2 \times 10^{-5}$ and $R_{b} \cong 2.8 \times 10^{-3}$. The analytical $b$ and $w$ fields shown in Fig. 2 depict a broad zone of ascent above the warm surface and a compensating zone of descent over the cold surface, roughly for $z<1.8 \mathrm{~m}$. In the upper part of these zones (at roughly $0.9 \mathrm{~m}<z<1.8 \mathrm{~m}$ ), adiabatic expansion/compression has reversed the senses of the buoyancy fields. Surprisingly, the numerical fields in the inhomogeneous INC- 1 and homogeneous HNC- 1 cases are very similar to each other and to the A-1 fields. The $u$ fields from A-1, INC-1, and HNC-1 shown in Fig. 3 are visually indistinguishable from one another.

To understand why the INC-1 and HNC-1 simulations are so similar, and to identify simulation parameters that might evince more substantial differences, we consider the idealized problem in which a specified buoyancy $b=$ $b_{0} e^{-\gamma z} \sin k x\left(\gamma=h^{-1}\right.$, where $h$ is the e-folding depth scale $)$ is the only forcing term in the Poisson equation $\nabla^{2} \Pi=$ $\partial b / \partial z$, with Neumann surface condition $\partial \Pi /\left.\partial z\right|_{0}=b(x, 0)$. This idealized problem is solved as

$\Pi_{\mathrm{INC}}^{*}=\frac{b_{0}}{\gamma^{2}-k^{2}}\left(k e^{-k z}-\gamma e^{-\gamma z}\right) \sin k x$.

The corresponding solution obtained with the homogeneous Neumann condition, $\partial \Pi /\left.\partial z\right|_{0}=0$, is 


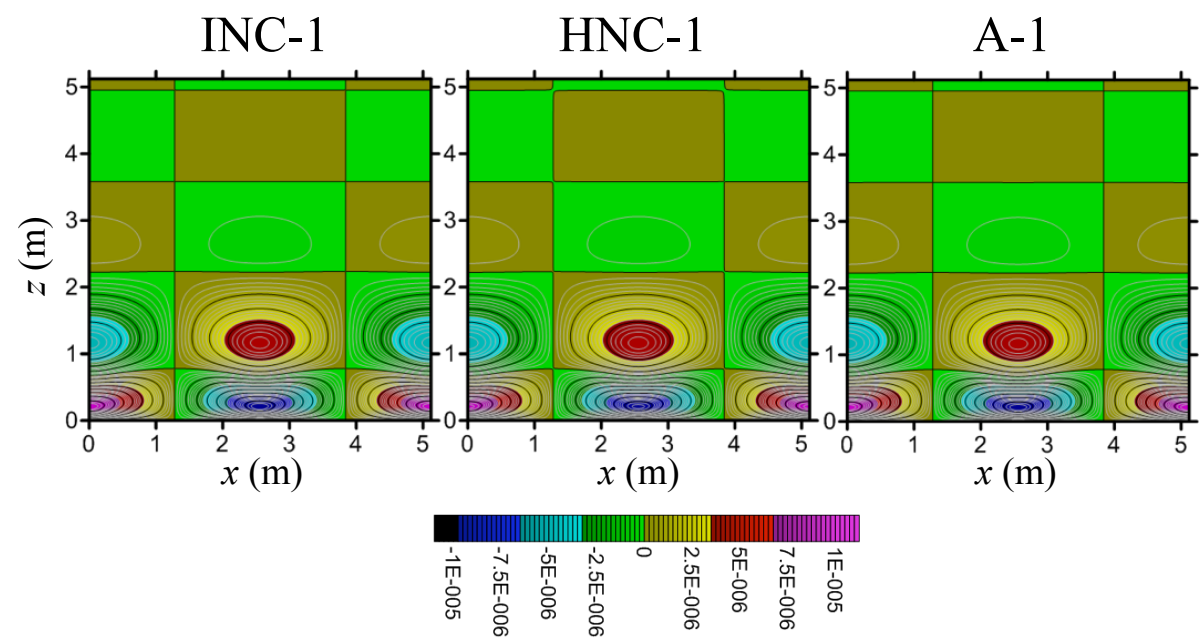

Figure 3. Vertical cross section of $u$ from the first test case. A-1 is the analytical solution. INC-1 is the numerical simulation with inhomogeneous Neumann condition for pressure. HNC-1 is the numerical simulation with the homogeneous Neumann condition for pressure. Colour bar units are $\mathrm{ms}^{-1}$.

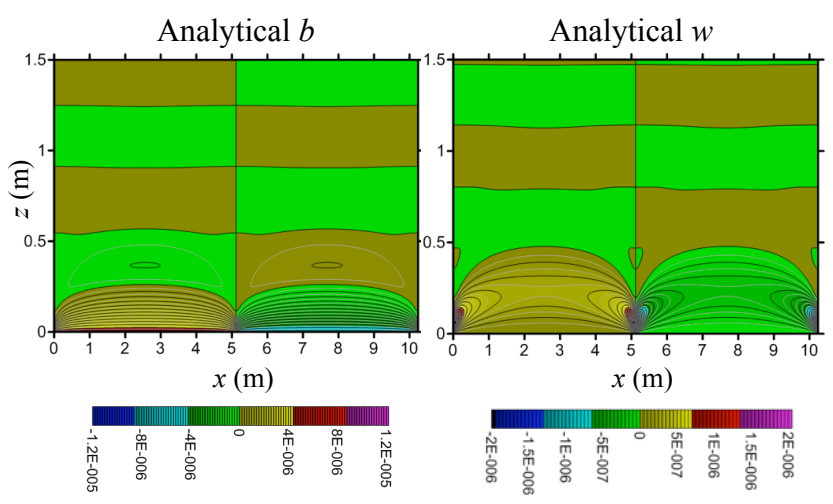

Figure 4. Vertical cross section of the analytical (A-2) buoyancy $b$ and vertical velocity $w$ fields from the second test case. Colour bar units are $\mathrm{ms}^{-2}$ for $b$, and $\mathrm{ms}^{-1}$ for $w$.

$\Pi_{\mathrm{HNC}}^{*}=\frac{b_{0}}{\gamma^{2}-k^{2}}\left(\frac{\gamma^{2}}{k} e^{-k z}-\gamma e^{-\gamma z}\right) \sin k x$.

The relative error $(\mathrm{RE})$ in the vertical pressure gradient force associated with Eqs. (44) and (45), defined as the local absolute error in that force divided by the local buoyancy, is calculated as

$\mathrm{RE} \equiv\left|\frac{\partial \Pi_{\mathrm{INC}}^{*} / \partial z-\partial \Pi_{\mathrm{HNC}}^{*} / \partial z}{b}\right|=e^{(a-1) k z}$,

where $a \equiv \gamma / k$. Written in terms of the depth scale $h$ and wavelength $\lambda=2 \pi / k, a$ can be interpreted as an aspect ratio characterizing the width to depth scales of the disturbance, $a=\lambda /(2 \pi h) \propto \lambda \gamma$. From Eq. (46) we see that RE decreases exponentially with $z$ for disturbances characterized by small aspect ratios, $a<1$ (which we refer to as deep disturbances),

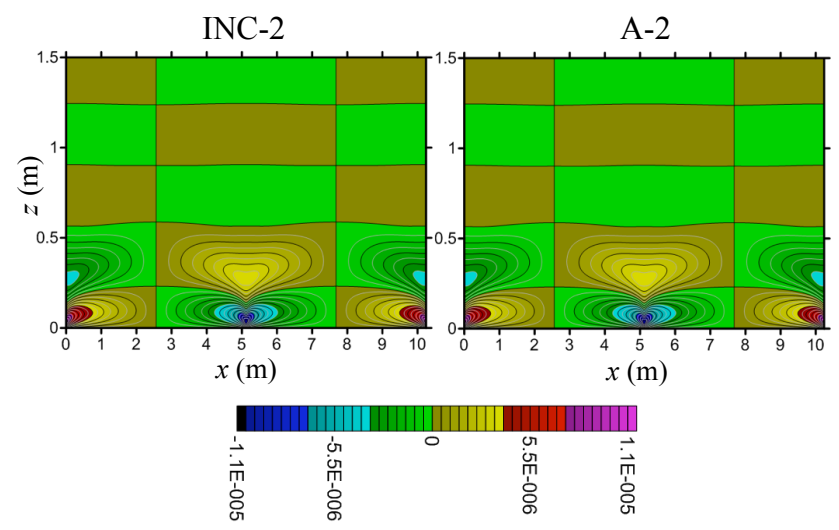

Figure 5. Vertical cross section of $u$ from the second test case. A-2 is the analytical solution. INC-2 is the numerical simulation with inhomogeneous Neumann condition for pressure. Colour bar units are $\mathrm{ms}^{-1}$.

and increases exponentially with $z$ for disturbances characterized by large aspect ratios, $a>1$ (which we refer to as shallow disturbances). The buoyancy in Fig. 2 is suggestive of $a<1$, which indicates that the first test could be classified as a deep (error-forgiving) simulation.

The preceding analysis suggests that simulations with shallow thermal disturbances $(a>1)$ might yield large differences between cases with inhomogeneous and homogeneous Neumann conditions. There did not appear to be a straightforward way to increase the effective $a$ by systematically varying the parameters (e.g. increasing $L$ tended to increase the effective $h$ ), but a set of suitable parameters were identified through trial and error and were used as the basis for the second test case. 


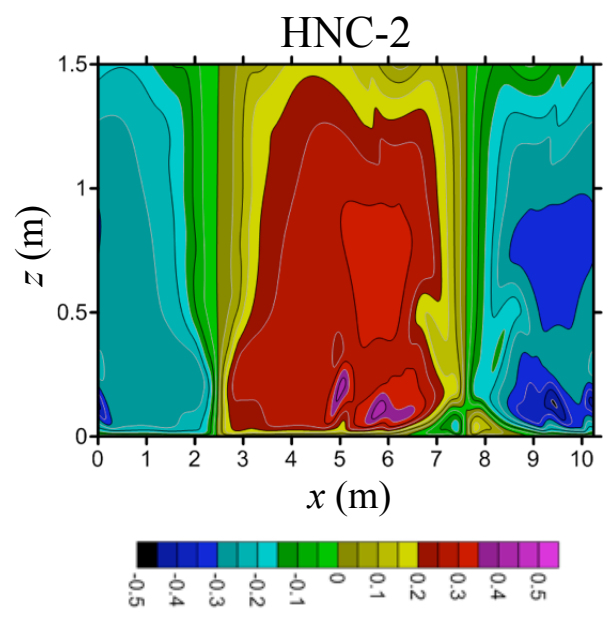

Figure 6. Vertical cross section of $u$ from HNC-2, the numerical simulation with homogeneous Neumann condition for pressure in the second test case. Colour bar units are $\mathrm{ms}^{-1}$.

In the second test, we set $v=\alpha=0.0001 \mathrm{~m}^{2} \mathrm{~s}^{-1}, N=$ $0.2 \mathrm{~s}^{-1}, L=10.24 \mathrm{~m}$, and $b_{\max }=5 \times 10^{-6} \mathrm{~m} \mathrm{~s}^{-2}$. The analytical solution A-2 was generated with 2049 points in the $x$ direction and 513 points in the $z$ direction, with grid spacings of $\Delta x=\Delta z=0.005 \mathrm{~m}$. The linearity criteria were satisfied with $R_{\eta} \cong 4.8 \times 10^{-5}$ and $R_{b} \cong 3.8 \times 10^{-3}$. In contrast to the counter-rotating convection rolls seen in the first test, the analytical $b$ and $w$ fields shown in Fig. 4 depict narrow updraft/downdraft pairs straddling the buoyancy discontinuities. Between the narrow updrafts is a broad region of relatively weak ascent. The $w$ and $b$ fields above the cold surface are mirror images of the fields above the warm surface. Note the change in the scales of the $x$ and (especially) the $z$ axes between Figs. 4 and 2: the low-level thermal disturbance in the second test is much shallower than the disturbance in the first test (and is suggestive of $a>1$ ). In this second test case we find dramatic differences between the inhomoge- neous INC-2 and homogeneous HNC-2 cases. Specifically, while the INC-2 and A-2 fields are in excellent agreement, the HNC-2 fields showed no signs of even approaching a steady state. Long after the INC-2 simulation had reached a steady state, the HNC-2 fields continued to amplify and develop asymmetric structures associated with flow nonlinearities. The very close agreement between the A-2 solution and the steady state in the INC-2 simulation is shown for the $u$ field in Fig. 5. The $u$ field in the disastrous HNC-2 simulation, at a time when a steady state had already been attained in the INC-2 simulation, is shown in Fig. 6.

\section{Summary}

The linearized Boussinesq equations for the motion of a viscous stably stratified fluid are solved analytically for a surface buoyancy that varies laterally as a square wave. The solution describes two-dimensional laminar convective structures such as thermal convective rolls and updraft/downdraft pairs. The main applications of the solution may be in code verification and the evaluation of different implementations of the surface pressure condition for the pressure Poisson equation. Tests have been conducted for cases where the aspect ratios of the thermal disturbance have been large and small. With attention restricted to disturbances of sufficiently small amplitude, the linear solution and numerically simulated fields with the inhomogeneous Neumann condition for pressure (which is appropriate in the context of the particular fractional step procedure adopted in our DNS code) have been found to be in excellent agreement for both tests. However, in tests with a mis-specified Neumann condition, an excellent agreement with the analytical solution has been found only for the deep (small aspect ratio) disturbance case; errors in the shallow (large aspect ratio) disturbance case have been catastrophic. 
Appendix A: Comment on the pressure condition at a lower solid surface

Consider a three-dimensional Boussinesq system with the equation of motion

$\frac{\partial \boldsymbol{u}}{\partial t}=-\nabla \Pi+v \nabla^{2} \boldsymbol{u}+\mathbf{F}$.

Here $\boldsymbol{u}=(u, v, w)$ is the three-dimensional velocity vector, $\Pi$ is a kinematic pressure perturbation, $v$ is the kinematic viscosity coefficient, and $\mathbf{F}$ is the sum of nonlinear acceleration and buoyancy terms. Applying the incompressibility condition,

$\nabla \cdot \boldsymbol{u}=0$

in the equation that results from taking the divergence of Eq. (A1) (e.g. Orszag et al., 1986) yields the Poisson equation,

$\nabla^{2} \Pi=\nabla \cdot \mathbf{F}$.

Although Eqs. (A1) and (A2) imply Eq. (A3a), the reverse statement is not generally true. Indeed, eliminating $\Pi$ from between Eq. (A3a) and the equation arising from taking the divergence of Eq. (A1) yields the diffusion equation $\partial \delta / \partial t=$ $\nu \nabla^{2} \delta$ for the velocity divergence $\delta \equiv \nabla \cdot \boldsymbol{u}$, whose solution is Eq. (A2) only if $\delta$ is 0 initially and on all boundaries (Orszag et al., 1986; Gresho and Sani, 1987; Vreman, 2014).

The same steps leading to Eq. (A3a) also lead to an alternative Poisson equation,

$\nabla^{2} \Pi=\nabla \cdot\left(v \nabla^{2} \boldsymbol{u}+\mathbf{F}\right)$

Although $\nabla \cdot v \nabla^{2} \boldsymbol{u}$ was omitted in Eq. (A3a) (this term is 0 if Eq. A2 is satisfied), without further constraints on $\delta$ (described above), Eq. (A2) may not be satisfied. Gresho and Sani (1987) showed that the retention of $\nabla \cdot v \nabla^{2} \boldsymbol{u}$ in Eq. (A3b) assures that Eq. (A2) is satisfied and thus leads to the paradox "If you include it, you don't need it; if you don't include it, you need it." Vreman (2014) revisited this paradox, and showed that for a standard staggered method, the discretized form of Eq. (A3b) is equivalent to that of Eq. (A3a) supplemented with the constraint that $\nabla \cdot \nabla^{2} \boldsymbol{u}=0$ $\left(\nabla^{2} \delta=0\right)$ on points adjacent to the solid boundary (with the same inhomogeneous Neumann boundary condition for $\Pi$ implied for Eqs. A3a and A3b). When supplemented with this $\nabla^{2} \delta=0$ near-wall condition, the diffusion equation for $\delta$ led to $\delta=0$ for all time. We note that Eq. (A3b) is the form adopted in our numerical code.

Evaluating the vertical component of Eq. (A1) on the surface, where the impermeability condition applies, yields the inhomogeneous Neumann condition,

$$
\left.\frac{\partial \Pi}{\partial z}\right|_{0}=\left.v \frac{\partial^{2} w}{\partial z^{2}}\right|_{0}+\left.F_{z}\right|_{0},
$$

where $w \equiv \boldsymbol{k} \cdot \boldsymbol{u}, F_{z} \equiv \boldsymbol{k} \cdot \mathbf{F}, \boldsymbol{k}$ is the upward unit vector, and ( ) $\left.\right|_{0}$ is a surface value. It has been argued that Eq. (A4), by itself, is not a proper boundary condition because it does not provide new information (it is not independent of the governing equations) and does not enforce the incompressibility condition (A2) at the boundary (Strikwerda, 1984; Henshaw, 1994; Sani et al., 2006). However, as pointed out by Henshaw (1994), many studies that impose Eq. (A4) (or a variant of it) also apply Eq. (A2) on the boundary.

In our numerical model, Eq. (A1) is integrated using a fractional step procedure with explicit treatment of the viscous term. First, a provisional velocity field $\widetilde{\boldsymbol{u}}$ that does not satisfy Eq. (A2) is obtained by integrating a discretized form of Eq. (A1) in which the pressure gradient is omitted. The provisional velocity is equal to the velocity at the end of the previous time step plus the sum of the forcing terms (nonlinear acceleration, buoyancy, and viscous stress) multiplied by the time step $\Delta t$. With the forcing terms explicitly evaluated, $\widetilde{\boldsymbol{u}}$ is readily computed throughout the flow domain, including on the surface, where, in surface-forced flows, the buoyancy will make a substantial contribution. In terms of $\widetilde{\boldsymbol{u}}$ and its vertical component $\widetilde{w}$, Eqs. (A3b) and (A4) become

$$
\begin{gathered}
\nabla^{2} \Pi=\frac{\nabla \cdot \widetilde{\boldsymbol{u}}}{\Delta t}, \\
\left.\frac{\partial \Pi}{\partial z}\right|_{0}-\left.\frac{1}{\Delta t} \widetilde{w}\right|_{0}=0 .
\end{gathered}
$$

In the second step, a velocity field that does satisfy Eq. (A2) is obtained by solving Eq. (A5) for $\Pi$ and then adding the pressure gradient force associated with $\Pi$ (multiplied by $\Delta t$ ) to $\widetilde{\boldsymbol{u}}$.

In some explicit fractional step procedures (including the DNS code used in our study), the problem of solving Eq. (A5) subject to Eq. (A6) with $\left.\widetilde{\boldsymbol{u}}\right|_{0}$ evaluated from model data is replaced by what appears to be an entirely different (but is actually equivalent) problem: solving Eq. (A5) subject to the homogeneous Neumann condition,

$$
\left.\frac{\partial \Pi}{\partial z}\right|_{0}=0
$$

in concert with $\left.\widetilde{\boldsymbol{u}}\right|_{0}$ being set to 0 , obviating the need to calculate $\left.\widetilde{\boldsymbol{u}}\right|_{0}$ from model data. It can be shown that $\left.\widetilde{w}\right|_{0}$ and the discretized form of $\partial \Pi /\left.\partial z\right|_{0}$ appear in the discretized form of Eq. (A5), valid half a grid point above the physical surface as $\partial \Pi /\left.\partial z\right|_{0}-\left.\widetilde{w}\right|_{0} / \Delta t$, that is, in the same combination as they appear in Eq. (A6). Thus, setting $\left.\widetilde{w}\right|_{0}$ and $\partial \Pi /\left.\partial z\right|_{0}$ to 0 is equivalent to implementing Eq. (A6) with the model-computed values of $\left.\widetilde{w}\right|_{0}$ : the discretized form of Eq. (A5) near the surface is the same in either case. Moreover, on the $\mathrm{C}$ grid, setting the tangential components $\left.\widetilde{u}\right|_{0}$ and $\left.\widetilde{v}\right|_{0}$ to 0 only affects the values of $\widetilde{u}$ and $\widetilde{v}$ half a grid point beneath the physical boundary. These values do not appear in the discretized form of Eq. (A5) at any $z$ level and thus have no bearing on the solution. In essence, the errors 
associated with the conflation of the two physically unjustifiable specifications (homogeneous Neumann condition for pressure, and $\left.\widetilde{\boldsymbol{u}}\right|_{0}=0$ ) cancel out. The homogeneous Neumann condition for pressure can be the source of confusion if the context in which the condition is applied is not made clear: it would be a correct condition if $\left.\widetilde{\boldsymbol{u}}\right|_{0}$ is set to 0 (per the equivalence described above), but it would be an incorrect condition if the explicit model-computed values of $\left.\widetilde{\boldsymbol{u}}\right|_{0}$ are used. In the experiments with the mis-specified condition described in Sect. 3, the homogeneous condition is imposed in the latter context. Unfortunately, in many numerical model descriptions, the nature of the surface pressure condition is left vague, for example, by not indicating whether a Neumann condition is homogeneous or inhomogeneous or, if a homogeneous Neumann condition is indicated, not mentioning how $\left.\widetilde{\boldsymbol{u}}\right|_{0}$ is treated.

Finally, we note that in fractional step procedures that treat the viscous term implicitly (e.g. Kim and Moin, 1985; Gresho, 1990; Armfield and Street, 2002; Guermond et al., 2006, and many others), the homogeneous Neumann con- dition is often applied as a surface condition for a Poisson equation, but it is again different from our implementation described in Sect. 3. In the implicit treatments, the provisional velocity is obtained as the solution of a boundary value problem ( $\left.\widetilde{\boldsymbol{u}}\right|_{0}$ should be specified; often it is set to 0$)$ in which the relevant Poisson equation resembles Eq. (A5) but applies to a scalar function (sometimes called a pseudo-pressure) that is not the real pressure. Temam (1991) refers to this scalar as, "... a technical quantity, a mathematical auxiliary ..." and advocates that it should not even be considered as an approximation of the pressure. Interestingly, in the context of implicit treatments, the homogeneous Neumann condition on the pseudo-pressure has sometimes been implicated as corrupting solution accuracy through the development of spurious numerical boundary layers adjacent to solid boundaries (Gresho, 1990; Guermond et al., 2006; Hosseini and Feng, 2011). 


\section{Code availability}

The Fortran program used to generate output data files from the analytical solution is available as a supplement to this article. That program (square.f) is configured for test A-1 but can be easily adjusted to run test A-2 or other tests. Running square.f automatically generates an output file for each dependent variable (e.g. u.dat) as well as an output file (square.out) that summarizes the test parameters and gives the computed values of the linearity ratios $R_{\eta}$ and $R_{b}$ defined in Eq. (43).

\section{The Supplement related to this article is available online at doi:10.5194/gmd-8-1809-2015-supplement.}

Acknowledgements. This research was supported by the National Science Foundation under grant AGS-1359698. Comments by Chiel van Heerwaarden, Juan Pedro Mellado, Inanc Senocak, and an anonymous reviewer are gratefully acknowledged.

Edited by: D. Lawrence

\section{References}

Armfield, S. and Street, R.: An analysis and comparison of the time accuracy of fractional-step methods for the Navier-Stokes equations on staggered grids, Int. J. Numer. Methods Fluids, 38, 255282, 2002.

Atkinson, B.: Meso-scale Atmospheric Circulations, Academic Press, Orlando, Florida, 495 p., 1981.

Axelsen, S. L., Shapiro, A., and Fedorovich, E.: Analytical solution for katabatic flow induced by an isolated cold strip, Environ. Fluid Mech., 10, 387-414, 2010.

Briggs, G. A.: Surface inhomogeneity effects on convective diffusion, Bound.-Lay. Meteorol., 45, 117-135, 1988.

Chandrasekhar, S.: Hydrodynamic and Hydromagnetic Stability, Oxford University Press, London, UK, 652 p., 1961.

Chorin, A. J.: Numerical solution of the Navier-Stokes equations, Math. Comput., 22, 745-762, 1968.

Egger, J.: On the linear two-dimensional theory of thermally induced slope winds, Beitr. Phys. Atmosph., 54, 465-481, 1981.

Fedorovich, E. and Shapiro, A.: Structure of numerically simulated katabatic and anabatic flows along steep slopes, Acta Geophys., 57, 981-1010, 2009a.

Fedorovich, E. and Shapiro, A.: Turbulent natural convection along a vertical plate immersed in a stably stratified fluid, J. Fluid Mech., 636, 41-57, 2009b.

Fedorovich, E., Nieuwstadt, F. T. M., and Kaiser, R.: Numerical and laboratory study of a horizontally evolving convective boundary layer. Part I: Transition regimes and development of the mixed layer, J. Atmos. Sci., 58, 70-86, 2001.

Gresho, P. M.: On the theory of semi-implicit projection methods for viscous incompressible flow and its implementation via a finite element method that also introduces a nearly consistent mass matrix. Part 1: Theory, Int. J. Numer. Methods Fluids, 11, 587620, 1990.
Gresho, P. M. and Sani, R. L.: On pressure boundary conditions for the incompressible Navier-Stokes equations, Int. J. Numer. Methods Fluids, 7, 1111-1145, 1987.

Guermond, J. L., Minev, P., and Shen, J.: An overview of projection methods for incompressible flows, Comput. Methods Appl. Mech. Engrg., 195, 6011-6045, 2006.

Hadfield, M. G., Cotton, W. R., and Pielke, R. A.: Large-eddy simulations of thermally forced circulations in the convective boundary layer. Part I: A small-scale circulation with zero wind, Bound.-Lay. Meteorol., 57, 79-114, 1991.

Henshaw, W. D.: A fourth-order accurate method for the incompressible Navier-Stokes equations on overlapping grids, J. Comput. Phys., 113, 13-25, 1994.

Hosseini, S. M. and Feng, J. J.: Pressure boundary conditions for computing incompressible flows with SPH, J. Comput. Phys., 230, 7473-7487, 2011.

Kang, S.-L., Lenschow, D., and Sullivan, P.: Effects of mesoscale surface thermal heterogeneity on low-level horizontal wind speeds, Bound.-Lay. Meteorol., 143, 409-432, 2012.

Kim, J. and Moin, P.: Application of a fractional-step method to incompressible Navier-Stokes equations, J. Comput. Phys., 59, 308-323, 1985.

Kundu, P. K.: Fluid Mechanics, Academic Press, San Diego, California, 638 p., 1990.

Mahrt, L., Sun, J., Vickers, D., MacPherson, J. I., Pederson, J. R., and Desjardins, R. L.: Observations of fluxes and inland breezes over a heterogeneous surface, J. Atmos. Sci., 51, 2484-2499, 1994.

McPherson, R. A.: A review of vegetation-atmosphere interactions and their influences on mesoscale phenomena, Prog. Phys. Geog., 31, 261-285, 2007.

Nordström, J., Mattsson, K., and Swanson, C.: Boundary conditions for a divergence free velocity-pressure formulation of the Navier-Stokes equations, J. Comput. Phys., 225, 874-890, 2007.

Orszag, S. A., Israeli, M., and Deville, M. O.: Boundary conditions for incompressible flows, J. Sci. Comput., 1, 75-111, 1986.

Patton, E. G., Sullivan, P. P., and Moeng, C.-H.: The influence of idealized heterogeneity on wet and dry planetary boundary layers coupled to the land surface, J. Atmos. Sci., 62, 2078-2097, 2005.

Petersson, N. A.: Stability of pressure boundary conditions for Stokes and Navier-Stokes equations, J. Comput. Phys., 172, 4070, 2001.

Pielke, R. A.: Influence of the spatial distribution of vegetation and soils on the prediction of cumulus convective rainfall, Rev. Geophys., 39, 151-177, 2001.

Rempfer, D.: On boundary conditions for incompressible NavierStokes problems, Appl. Mech. Rev., 59, 107-125, 2006.

Sani, R. L., Shen, J., Pironneau, O., and Gresho, P. M.: Pressure boundary condition for the time-dependent incompressible Navier-Stokes equations, Int. J. Numer. Methods Fluids, 50, 673-682, 2006.

Segal, M. and Arritt, R. W.: Non-classical mesoscale circulations caused by surface sensible heat-flux gradients, B. Am. Meteorol. Soc., 73, 1593-1604, 1992.

Shapiro, A. and Fedorovich, E.: Similarity models for unsteady free convection flows along a differentially cooled horizontal surface, J. Fluid Mech., 736, 444-463, 2013.

Shapiro, A. and Fedorovich, E.: A boundary-layer scaling for turbulent katabatic flow, Bound.-Lay. Meteorol., 153, 1-17, 2014. 
Shirokoff, D. and Rosales, R. R.: An efficient method for the incompressible Navier-Stokes equations on irregular domains with no-slip boundary conditions, high order up to the boundary, J. Comput. Phys., 230, 8619-8646, 2011.

Simpson, J. E.: Sea Breeze and Local Winds, Cambridge University Press, Cambridge, UK, 234 p., 1994.

Strikwerda, J. C.: Finite difference methods for the Stokes and Navier-Stokes equations, SIAM J. Sci. Stat. Comput., 5, 56-68, 1984.
Temam, R.: Remark on the pressure boundary condition for the projection method, Theoret. Comput. Fluid Dynam., 3, 181-184, 1991.

van Heerwaarden, C. C., Mellado, J. P., and de Lozar, A.: Scaling laws for the heterogeneously heated free convective boundary layer, J. Atmos. Sci., 71, 3975-4000, 2014.

Vreman, A. W.: The projection method for the incompressible Navier-Stokes equations: The pressure near a no-slip wall, J. Comput. Phys., 263, 353-374, 2014. 